\title{
Mechanically Robust Gels Formed from Hydrophobized Cellulose Nanocrystals
}

\author{
Rinat Nigmatullin, ${ }^{* \dagger}{ }^{\dagger}$ Robert Harniman, ${ }^{\ddagger}$ Valeria Gabrielli, ${ }^{\S}$ Juan C. Muñoz-García, ${ }^{\S}$ \\ Yaroslav Z. Khimyak, ${ }^{\S}$ Jesús Angulo, ${ }^{\S}$ and Stephen J. Eichhorn*,† \\ ${ }^{\dagger}$ Bristol Composites Institute (ACCIS), University of Bristol, Bristol BS8 1TR, United Kingdom \\ ${ }^{\ddagger}$ School of Chemistry, University of Bristol, Bristol BS8 1TS, United Kingdom \\ ${ }^{\S}$ School of Pharmacy, University of East Anglia, Norwich Research Park, Norwich NR4 7TJ, United Kingdom
}

\author{
Supporting Information
}

\begin{abstract}
Cellulose nanocrystals (CNCs) that bind to each other through associative hydrophobic interactions have been synthesized by modifying sulfated CNCs (sCNCs) with hydrophobic moieties. These octyl-CNCs form gels at significantly lower concentrations than parent sCNCs, producing extremely strong hydrogels. Unlike sCNCs, these octyl-CNCs do not form ordered liquid crystalline phases indicating a random association into a robust network driven by hydrophobic interactions. Furthermore, involvement of the octyl-CNCs into multicomponent supramolecular assembly was demonstrated in combination with starch. AFM studies confirm favorable interactions between starch and octyl-CNCs, which is thought to be the source of the dramatic increase in gel strength.
\end{abstract}

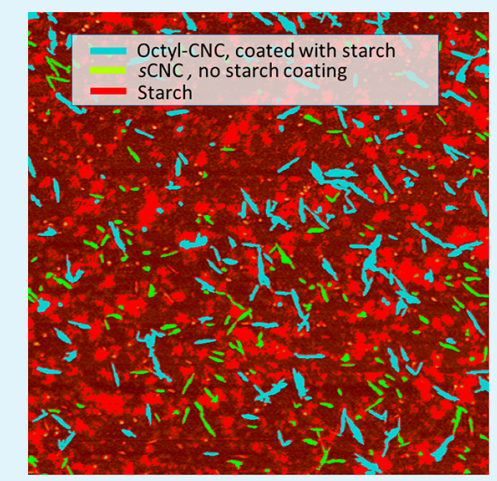

KEYWORDS: cellulose nanocrystals (CNCs), gels, starch, rheology, adhesive force

$\mathrm{T}$ he formation of strong gels from cellulose nanofibrils is well-documented in the literature, and are potentially useful as rheological modifiers in food, paints, and in homecare and beauty products. ${ }^{1-3}$ Their stability in each of these application environments is predicated on their mechanical integrity. Cellulose nanocrystals (CNCs), a form of highly crystalline rodlike cellulose, ${ }^{4}$ are also reported to form gels. ${ }^{5-7}$ It is generally agreed that rheological properties of CNC suspensions are defined by their ability to form biphasic systems of isotropic and chiral nematic phases. ${ }^{5,6,8-10}$ At low concentrations, CNC suspensions are practically isotropic and behave almost as Newtonian fluids. With an increase in the $\mathrm{CNC}$ concentration, these suspensions exhibit the properties of viscoelastic fluids due to the formation and growing fraction of the liquid crystal phase. Further increases in concentration lead to the formation of randomly entangled gels with dominating elastic properties. Typically, these gels are relatively weaker than those formed of fibrils due to relatively fewer interfiber crossings. Critical concentrations at the transition between these states of $\mathrm{CNC}$ suspensions depend on their aspect ratio and surface charge, and the ionic strength of the aqueous media. CNCs have been explored as reinforcing additives for hydrogels both as a component of supramolecular hydrogels ${ }^{11-13}$ and a reactive component incorporated into polymer networks via covalent binding. ${ }^{14-16}$ Herein we report the formation of extremely strong gels based on CNCs using chemical modification of their surface to introduce hydrophobic alkyl chains on their surface (octyl-CNCs). These strong gels form at much lower concentrations than typical CNC materials, which holds potential for their use for a wide range of applications where stability is required. Moreover, we demonstrate that octyl-CNCs induce the formation of strong supramolecular hydrogels in combination with starch. The work represents a new form of nanocellulose based colloidal or hybrid supramolecular and colloidal hydrogel. Preservation of the anionic charges of parent CNCs enables high water intake while the addition of hydrophobic groups enhances network formation via the fibrils, self-association or supramolecular binding of water-soluble polymers.

Modified CNCs were synthesized from materials produced by hydrolysis with sulfuric acid. From here we denote the initial CNCs as $s \mathrm{CNCs}$ to indicate that their surface is functionalized with sulfate half-ester groups. Octyl-CNCs were synthesized in two steps: periodate oxidation of $s \mathrm{CNCs}$ followed by the reductive amination reaction between aldehyde groups of the oxidized $s$ CNCs and octylamine. The modification of the CNCs with octyl chains has been confirmed by FTIR and NMR (Figures S2 and S3). From the analysis of ${ }^{1} \mathrm{H}-{ }^{13} \mathrm{C} \mathrm{CP}$ MAS NMR spectra the degree of surface functionalization was found to be around 4\% (Table S1). Both sCNCs and octylCNCs were used in the sodium form as never dried materials.

Received: March 29, 2018

Accepted: May 23, 2018

Published: May 23, 2018 
The viscoelastic properties of octyl-CNCs and $s$ CNCs suspensions over a wide range of concentration are compared in Figure 1. In contrast to $s \mathrm{CNCs}, G^{\prime}$ is found to be higher than

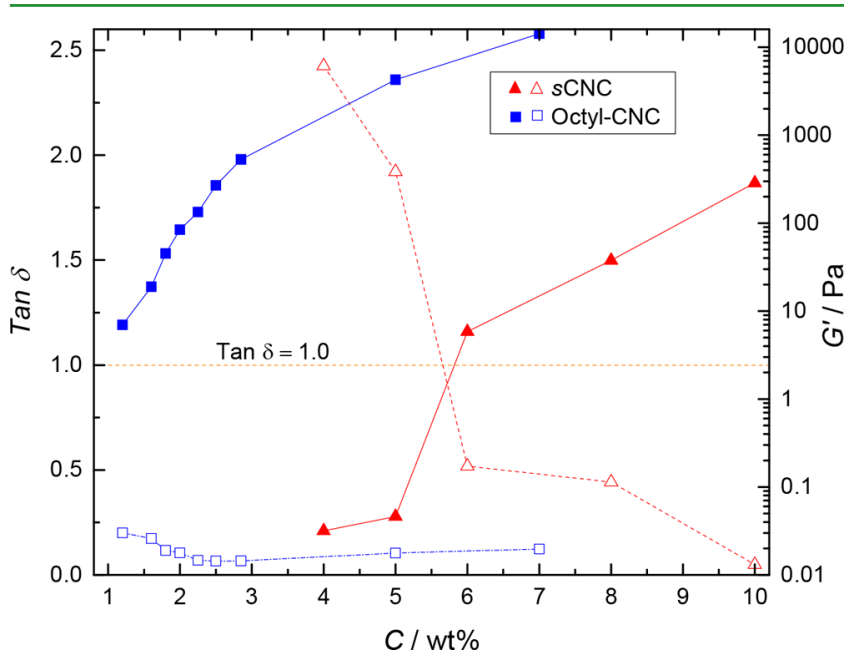

Figure 1. Storage modulus $\left(G^{\prime}\right)$ (filled symbols) and $\tan \delta$ (open symbols) as a function of CNC concentration $(C)$ at an angular frequency of $6.34 \mathrm{rad} \mathrm{s}^{-1}$ and strain $1.5 \%$. The dotted line represents the point at which $\tan \delta=1$. When $\tan \delta<1$ then $G^{\prime}>G^{\prime \prime}$.

$G^{\prime \prime}(\tan \delta<1)$ for octyl-CNCs over the whole range of concentrations, showing the dominance of elastic properties. At concentrations above $2.0 \mathrm{wt} \%$ octyl-CNCs formed strong selfsupporting gels with a value of $G^{\prime}$ larger than $G^{\prime \prime}$; by a factor of 3 or more. Suspensions of octyl-CNCs however did not form invertible gels at concentrations below $2.0 \mathrm{wt} \%$ (Figure S4) and there was less than a decade difference in $G^{\prime}$ and $G^{\prime \prime}$ for a 1.2 wt $\%$ suspension $(\tan \delta>0.18)$. These values are characteristic for "weak gels" or "structured fluids" which are usually formed by a tenuous association of mesoscopic domains.

Naturally $G^{\prime}$ increased with an increase in concentration for both types of CNCs. However, for suspensions of the same concentration, $G^{\prime}$ is significantly higher for octyl-CNCs. For example, at 5 wt \% $G^{\prime}$ for the octyl-CNCs gel is almost 5 orders of magnitude higher than for $s$ CNCs; c.f. 4300 and $0.05 \mathrm{~Pa}$, respectively. Moreover, in contrast to octyl-CNCs, $\tan \delta$ is strongly dependent on the concentration of the $s \mathrm{CNCs}$ suspension. The storage modulus also increased significantly for the $s \mathrm{CNC}$ suspension, and $\tan \delta$ only fell below 1.0 when concentrations were higher than 5 wt \%. Thus, the elastic properties of $s \mathrm{CNC}$ suspensions only became significant when concentrations exceeded 5 wt $\%$.

In line with results of the oscillatory measurements, steadyshear experiments demonstrated a significant increase in the viscosity of the octyl-CNC suspension/gels compared to $s \mathrm{CNCs}$, as shown in Figure 2. Previously a three-region pattern of viscosity dependence on concentration has been reported for CNCs. ${ }^{5,6,10,17}$ Such a response has been previously attributed to the transformation of $\mathrm{CNC}$ dispersions from an isotropic system to biphasic systems of isotropic and liquid crystal phases, and finally to a gel state. Similar patterns of viscosity changes with concentration are noted for octyl-CNCs. However, for octyl-CNCs a sharp increase in viscosity was observed between 1.2 and 2.8 wt \%. For the $s \mathrm{CNCs}$, the sharp increase was observed between 5 and 8 wt \%. Notably, octyl$\mathrm{CNC}$ suspensions/gels are not birefringent over the whole
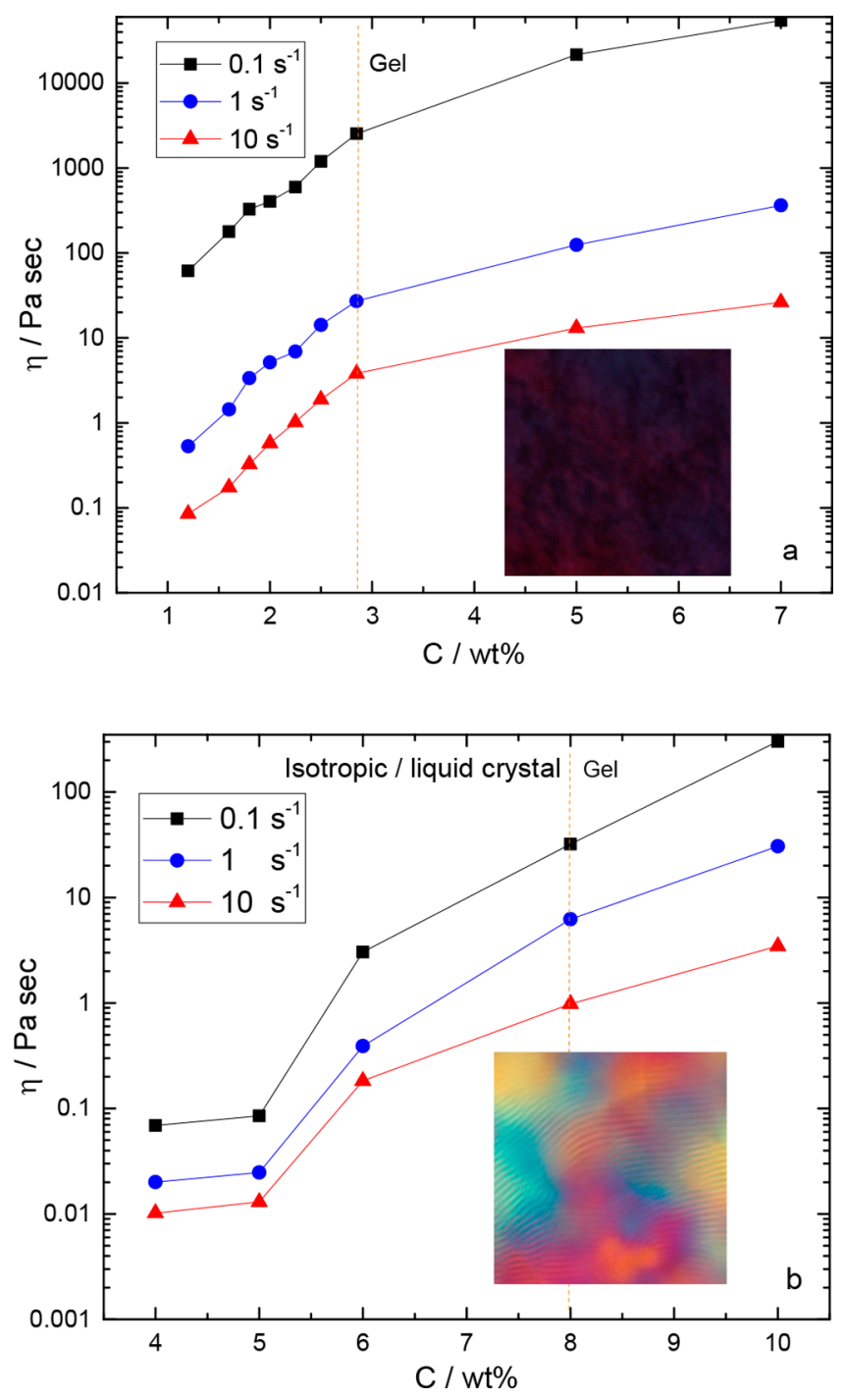

Figure 2. Typical dependence of steady flow viscosity $(\eta)$ of (a) octylCNCs and (b) $s$ CNCs suspensions in deionized water at three different shear rates: $0.1,1$, and $10 \mathrm{~s}^{-1}$ as a function of CNC concentration $(C)$. Insets: polarized optical micrographs of 5 wt $\%$ octyl-CNCs and $s \mathrm{CNCs}$ gels.

concentration range. Inserts in Figure 2 demonstrate the formation of a characteristic texture for a chiral nematic phase of the 5 wt \% $s$ CNCs suspension. An octyl-CNC gel of the same concentration is featureless under polarized light, indicating the lack of a chiral nematic phase. Thus, it appears that octyl-CNCs lose the ability to form long-range ordered structures. Instead, hydrophobic interactions are thought to drive their association into a network which induces the formation of a gel state with increased strength and viscosity. Enhanced rheological properties have been reported for other hydrophobized nanocelluloses, such as nanofibrillated cellulose with grafted synthetic hydrophobic polymers. ${ }^{18}$ Random association of octyl-CNCs and their gelation at low concentrations prevents the formation of a liquid crystalline phase. Gelation at lower concentrations was assumed previously to be the cause for the inhibition of the ordered structure formation of cationically modified CNCs. ${ }^{19}$

Formation of stronger networks involving octyl-CNCs are expected not to be limited by self-association. Strongly associative CNCs can be used for assembling supramolecular 
hydrogels in multicomponent systems. Here we test this concept to modify the gelation of starch-a common gelling and pasting agent in foodstuffs. Amylose, a linear component of starch, is known to form inclusion complexes with hydrophobic and amphiphilic ligands such as fatty acids, lactones, aldehydes, etc. $^{20}$ In such complexes a hydrophobic cavity of a single amylose helix accommodates a hydrophobic guest or hydrophobic part of an amphiphilic ligand. Inclusion complexes of large ligands such as hydrophobic synthetic polymers ${ }^{21,22}$ or single-walled carbon nanotubes (SWCNTs) ${ }^{23}$ have been also assembled with amylose.

Starch properties vary depending on the source. However, starches of any source are known to form gels only at relatively high concentrations (above $6 \mathrm{wt} \%)^{24,25}$ We attempted to achieve gelation in starch/CNC systems, when individual concentrations are lower than those required for the formation of invertible gels. Figure 3 compares the rheological properties

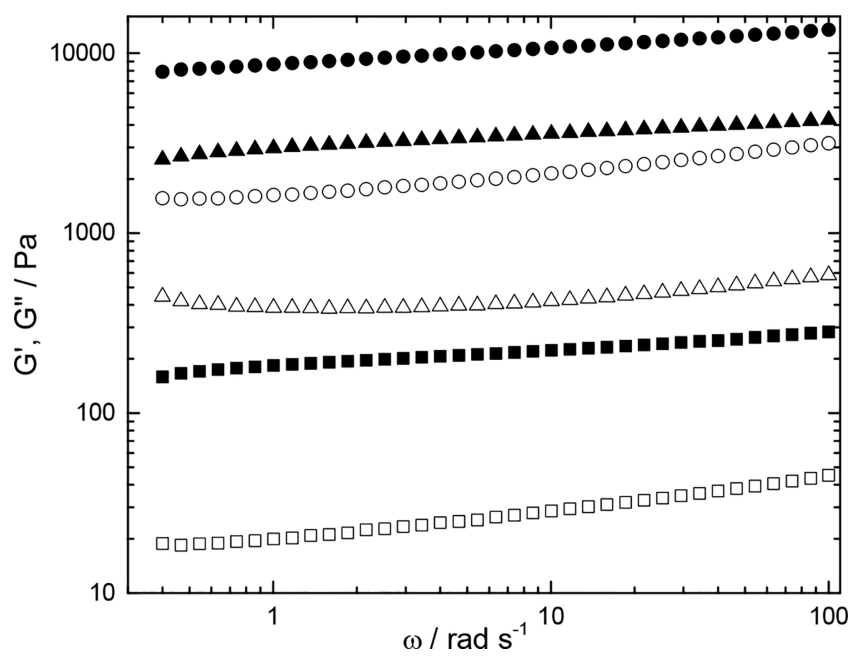

Figure 3. Typical mechanical spectra of starch/CNC gels $\left(G^{\prime}\right.$, filled symbols; $G^{\prime \prime}$, open symbols). 2 wt $\%$ starch $/ 2$ wt $\% s C N C$ in $0.1 \mathrm{M}$ $\mathrm{KCl}(\mathbf{\square}, \square) ;$,2 wt \% starch $/ 2$ wt \% octyl-CNC in $0.1 \mathrm{M} \mathrm{KCl}(\bullet, \bigcirc)$; 2 wt $\%$ starch $/ 2$ wt $\%$ octyl-CNC in deionized water $(\boldsymbol{\Delta}, \triangle)$.

of gels prepared with $s$ CNCs and octyl-CNCs. Both gels showed a weak dependence on frequency, responding as solidlike materials. However, starch/octyl-CNCs gels had almost 50 times higher values of the storage modulus $\left(G^{\prime}\right)$ compared to the starch $/ s \mathrm{CNC}$ gels. The value of $G^{\prime}$ for the $\operatorname{starch} / s \mathrm{CNCs}$ system is marginally higher than $s \mathrm{CNCs}$ alone at a 2 wt \% concentration (210 and $110 \mathrm{~Pa}$ respectively at frequency 6.34 $\mathrm{rad} \mathrm{s}^{-1}$ ). However, $G^{\prime}$ increases from $80 \mathrm{~Pa}$ for a $2 \mathrm{wt} \%$ octylCNC suspension to $1.02 \times 10^{4} \mathrm{~Pa}$ in the starch octyl-CNCs system. The presence of electrolyte further improved the elastic properties of starch/octyl-CNC gels. A 3-fold increase in $G^{\prime}$ of starch/octyl-CNC gels was observed for the gel prepared in 0.1 $\mathrm{M} \mathrm{KCl}$ (Figure 3). Such changes indicate strong interactions between starch and hydrophobically modified CNCs. On the contrary, a lack of association between $s \mathrm{CNCs}$ and starch due to a poor interaction between these polysaccharides inhibits the formation of a network.

AFM studies have provided further insight into starch/CNCs interactions. A number of studies involving high-resolution imaging techniques such as AFM and TEM have demonstrated that the majority of CNCs are not single crystals but lateral assemblies of several (2 to 4) elementary crystals. ${ }^{26,27}$ Deposited on the surface of freshly cleaved mica these assemblies tend to lie flat. As a result, AFM height measurements enable a precise determination of the width of the elementary crystal. Therefore, CNC height imaging provides a reliable tool for evaluating the possible buildup of material upon a CNC surface. ${ }^{27}$ AFM images of the topology of starch/CNCs mixtures deposited from the diluted suspensions revealed shapeless patches spreading from the surface of octylCNCs (Figure 4a, top row). Height profiles taken along the length of the isolated octyl-CNC nanorods (Figure S5a) demonstrate small variations in the diameters (average height $=$ $6.9 \pm 0.9 \mathrm{~nm}$ ). This indicates the formation of a uniform starch layer on the surface of the octyl-CNCs. Considering that diameter of our octyl-CNCs is $\sim 4 \mathrm{~nm}$, the thickness of the starch layer on the surface is thought to be $\sim 1.5 \mathrm{~nm}$.

However, there is no gain in the height of $s \mathrm{CNCs}$ in the presence of starch (average height remains at $4.1 \pm 0.6 \mathrm{~nm}$, Figure S5a). This suggests that starch is not adhering to the surface of the $s \mathrm{CNCs}$. The lack of starch affinity to $s \mathrm{CNC}$ is even more evident from AFM adhesion imaging (Figure 4a, bottom row). There are striking differences in the AFM probe adhesion response toward the $s \mathrm{CNCs}$ and octyl-CNCs in a mixture with starch. $s \mathrm{CNCs}$ appear significantly darker in the adhesion images compared with both octyl-CNCs and shapeless starch patches surrounding the CNCs. Thus, the AFM tip exhibits a significantly weaker adherence to $s \mathrm{CNC}$ nanorods compared with octyl-CNCs and starch. It appears therefore that starch does not adhere to the $s \mathrm{CNCs}$. In contrast, the adhesion mapping for octyl-CNCs indicates that these hydrophobized materials are coated with starch, since their color shade is similar to the surrounding starch patches. This observation is in line with topography images which showed an increase in the octyl-CNC diameter due to an interaction with starch.

Following on from these qualitative observations, AFM probe adhesion responses toward various surfaces were quantified; samples containing bare $s \mathrm{CNC}$, bare octyl-CNC and both CNCs in the presence of starch were assessed. Figure $4 \mathrm{~b}$ represents the differences in adhesive responses of the silicon AFM tip toward different materials compared with the tip adhesion to mica. Normalization of adhesive interaction forces to the force of tip interaction with the background mica allows a simple comparison of results obtained with different tips and substrates as adhesive interaction forces can prove complex in ambient environments and are typically dominated by the formation of a capillary bridge. ${ }^{28}$ In such a way, each sample was characterized using a fresh cantilever which ensures sharp tips (maintaining a tip radius of $\sim 2 \mathrm{~nm}$ for different samples) for maximum image resolution and tip surfaces conditioned in the same environment for comparative force measurements.

As can be seen from Figure $4 \mathrm{~b}$, bare $s \mathrm{CNC}$ and octyl-CNC significantly differ in their affinity toward the AFM probe; relative adhesive responses of $24 \pm 5 \%$ and $69 \pm 8 \%$ were respectively obtained. AFM probe adhesion to starch (42 \pm $7 \%)$ is lower than adhesion to bare octyl-CNCs but higher than to bare $s$ CNCs. Mixing starch with octyl-CNCs leads to a significant drop in the AFM probe adhesion to octyl-CNCs (38 $\pm 6 \%)$. In fact, adhesion between the probe and octyl-CNCs in a starch/octyl-CNC mix is the same as the probe/starch adhesion within their respective error bounds (cf. $38 \pm 6 \%$ and $42 \pm 7 \%$ ). This further verifies that octyl-CNCs are coated with starch. However, the presence of starch does not influence the interaction between the $s \mathrm{CNCs}$ and the AFM probe (relative 


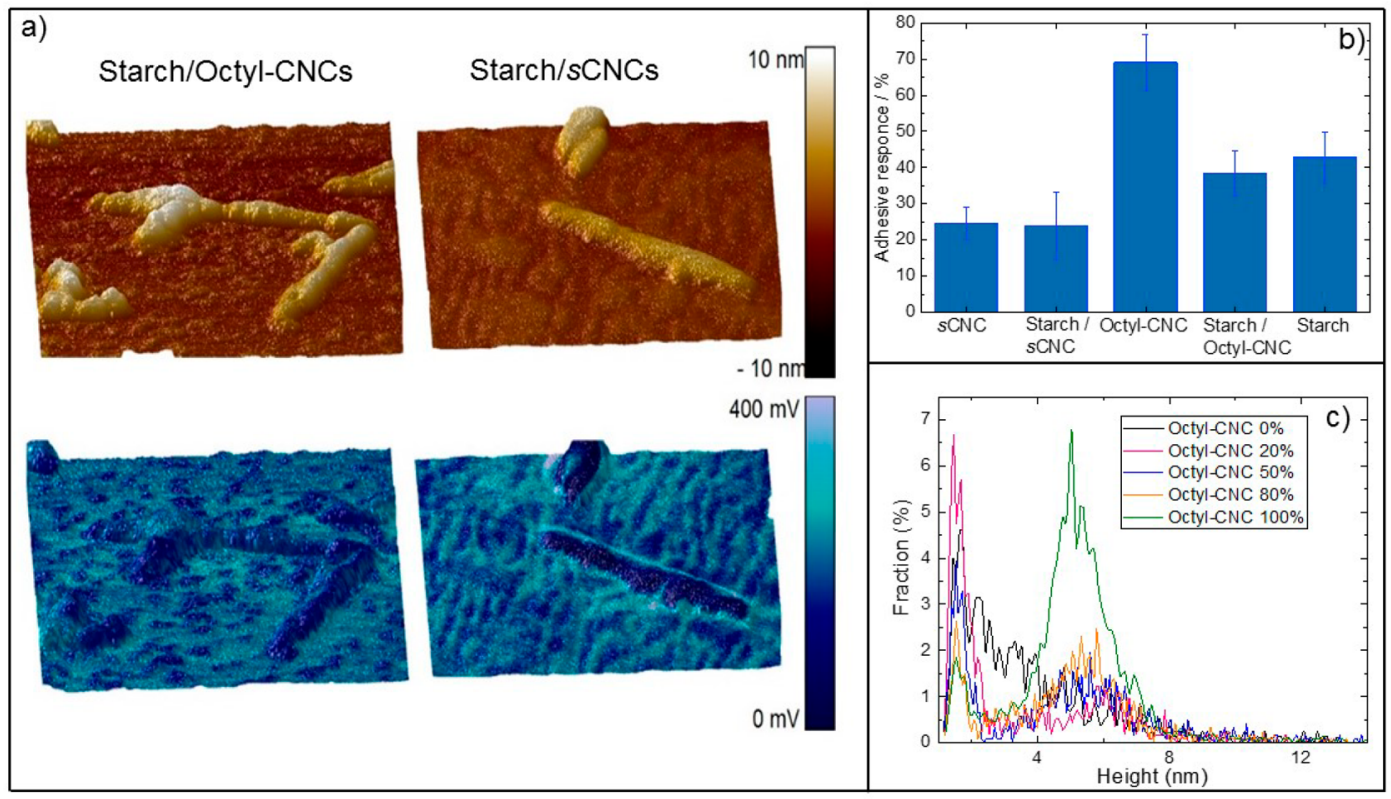

Figure 4. AFM study of starch/CNC systems: (a) typical AFM height (top row) and adhesion (bottom row) images for mixtures of starch and CNCs deposited on freshly cleaved mica using suspensions with a total solid content of $\sim 5 \mathrm{mg} / \mathrm{mL}$ (starch/CNC mass ratio $1.5 / 1$; scan size $300 \times$ $300 \mathrm{~nm}$ ); (b) relative difference in the adhesive response of the AFM probe against starch, $s \mathrm{CNCs}$, octyl-CNCs, and both type of CNCs in starch/ CNCs systems with respect to mica; (c) distribution of relative frequency of particle height based on AFM topographic images $(4 \mu \mathrm{m} \times 4 \mu \mathrm{m})$ of starch combined with mixtures of CNCs with octyl-CNC content $0,20,50,80$, and $100 \%$ (starch to CNC ratio 1.5 to 1 ).

adhesion $24 \pm 9 \%$ compared to $24 \pm 5 \%$ for bare $s \mathrm{CNC})$. This confirms the lack of affinity between starch and $s \mathrm{CNCs}$ and $s \mathrm{CNCs}$ remain uncoated when mixed with starch. Thus, AFM allows clear identification of different types of CNCs even when they are combined with starch.

To further asses the effect of supramolecular association of different types of CNCs on the properties of starch/CNC gels, a series of systems were produced with a mix of both hydrophobized and nonhydrophobized CNCs. It was found that $s \mathrm{CNCs}$ weakened interactions, thereby decreasing the elastic properties of starch/CNC gels (Figure S6a). Replacing $25 \%$ of octyl-CNCs with $s$ CNCs caused an almost 15 times decrease in $G^{\prime}$, despite maintaining the same content of rigid CNCs. In line with the results on the elastic properties of starch/CNC systems, steady flow experiments revealed significant increases in the viscosity of starch/octyl-CNC gels compared with starch/sCNC systems (Figure S6b). The flow curves for starch/CNC gels showed short intervals of a Newtonian plateau at low shear rates. The apparent viscosity in this region increased from $7 \mathrm{kPa}$ s for gels containing $s \mathrm{CNC}$ to $210 \mathrm{kPa}$ s for hydrogels with octyl-CNCs. Similar to the storage modulus, the viscosity decreased for gels containing mixtures of $s \mathrm{CNC}$ and octyl-CNCs. At high shear rates, the flow curves tended to overlap, possibly reflecting the fact that the gels contained the same amounts of starch and CNCs.

Combining $s \mathrm{CNCs}$ and octyl-CNCs with starch allows the preparation of gels with a broad range of rheological properties (Figure S7). A weakening effect of $s \mathrm{CNCs}$ on starch/octyl$\mathrm{CNCs}$ was observed even when the content of octyl-CNCs was kept at 2 wt \%. Despite the increase in the total CNC content, starch/CNC gels had a lower yield stress and viscosity when an additional $s$ CNC content $(25$ and 50 wt \% with respect to octyl-CNC) was added to the gels. In fact, gels with a higher $\mathrm{CNC}$ content ( $3 \mathrm{wt} \%$ ) were weaker than gels containing $2 \mathrm{wt}$ $\%$ octyl-CNCs or 2 wt \% octyl-CNCs with 0.5 wt \% sCNCs.
Thus, different combinations of $s \mathrm{CNCs}$ and octyl-CNCs allow the tuning of rheological properties of gels according to a desired application.

Poor starch $/ s \mathrm{CNC}$ interactions and lack of $s \mathrm{CNC}$ integration into a multicomponent supramolecular network was confirmed by the particle height analysis of the topographic images of starch combined with both sCNCs and octyl-CNCs (Figure $4 c)$. An increase in the octyl-CNC content causes a drop in number of thin (less than $3 \mathrm{~nm}$ ) particles, which are loose starch particles in the starch/CNC mixtures. Thus, a growing fraction of starch adheres to CNC surfaces when the octylCNCs content increases. Simultaneously, thicker particles become dominant with an increase in octyl-CNC content. These particles represent octyl-CNCs coated with starch reflecting redistribution of the loose starch to octyl-CNCbound states.

In summary, we have produced gels using hydrophobically modified CNCs. These gels show very high storage moduli compared to nonfunctionalized $s$ CNCs. Associative hydrophobic CNCs form gels at lower concentrations. Furthermore, it is shown that surface functionalization increases the affinity of CNCs to starch, enabling the formation of strong gels at relatively low concentrations. Hydrophobized CNCs are expected to advance rheological modifying and gel forming formulations for food, cosmetic, and pharmaceutical products. Materials based on hydrophobic CNCs could be further diversified in combination with other hydrocolloids such as starch and other polysaccharides. Hydrophobically derivatized CNCs can enable further progress in polymer nanocomposites due to potentially enhanced compatibility with hydrophobic polymer matrices. Specifically, the demonstrated high affinity between octyl-CNC and starch can pave the way to composites combined with thermoplastic starch. 


\section{ASSOCIATED CONTENT}

\section{S Supporting Information}

The Supporting Information is available free of charge on the ACS Publications website at DOI: 10.1021/acsami.8b05067.

Synthesis, experimental details, FTIR, NMR results, optical images of gel formation, determination of $\mathrm{CNC}$ sizes from AFM, rheology results for starch/CNC gels (PDF); all data from this paper can also be found on the University of Bristol repository at https://data.bris.ac.uk/ data/ (PDF)

\section{AUTHOR INFORMATION}

\section{Corresponding Authors}

*E-mail: rn17541@bristol.ac.uk (R.N.).

*E-mail: s.j.eichhorn@bristol.ac.uk (S.J.E.).

\section{ORCID $\odot$}

Stephen J. Eichhorn: 0000-0003-4101-273X

\section{Author Contributions}

The manuscript was written through contributions of all authors led by R.N. and S.J.E. All authors have given approval to the final version of the manuscript.

\section{Funding}

The Engineering and Physical Sciences Research Council (EPSRC) is acknowledged for provision of financial support (EP/N03340X/2, EP/N033337/1). PeakForce atomic force microscopy was carried out in the Chemical Imaging Facility, University of Bristol with equipment funded by EPSRC under Grant "Atoms to Applications" (EP/K035746/1).

\section{Notes}

The authors declare no competing financial interest.

\section{ACKNOWLEDGMENTS}

We are grateful for the use of the University of East Anglia (UEA) Faculty of Science NMR facility.

\section{REFERENCES}

(1) Saito, T.; Uematsu, T.; Kimura, S.; Enomae, T.; Isogai, A. Selfaligned integration of native cellulose nanofibrils towards producing diverse bulk materials. Soft Matter 2011, 7 (19), 8804-8809.

(2) Pääkkö, M.; Ankerfors, M.; Kosonen, H.; Nykänen, A.; Ahola, S.; Österberg, M.; Ruokolainen, J.; Laine, J.; Larsson, P. T.; Ikkala, O.; Lindström, T. Enzymatic Hydrolysis Combined with Mechanical Shearing and High-Pressure Homogenization for Nanoscale Cellulose Fibrils and Strong Gels. Biomacromolecules 2007, 8 (6), 1934-1941.

(3) Nordenström, M.; Fall, A.; Nyström, G.; Wågberg, L. Formation of Colloidal Nanocellulose Glasses and Gels. Langmuir 2017, 33 (38), 9772-9780.

(4) Habibi, Y.; Lucia, L. A.; Rojas, O. J. Cellulose Nanocrystals: Chemistry, Self-Assembly, and Applications. Chem. Rev. 2010, 110 (6), 3479-3500.

(5) Shafiei-Sabet, S.; Hamad, W. Y.; Hatzikiriakos, S. G. Rheology of Nanocrystalline Cellulose Aqueous Suspensions. Langmuir 2012, 28 (49), 17124-17133.

(6) Ureña-Benavides, E. E.; Ao, G.; Davis, V. A.; Kitchens, C. L. Rheology and Phase Behavior of Lyotropic Cellulose Nanocrystal Suspensions. Macromolecules 2011, 44 (22), 8990-8998.

(7) Lewis, L.; Derakhshandeh, M.; Hatzikiriakos, S. G.; Hamad, W. Y.; MacLachlan, M. J. Hydrothermal Gelation of Aqueous Cellulose Nanocrystal Suspensions. Biomacromolecules 2016, 17 (8), 27472754.

(8) Oguzlu, H.; Danumah, C.; Boluk, Y. Colloidal behavior of aqueous cellulose nanocrystal suspensions. Curr. Opin. Colloid Interface Sci. 2017, 29, 46-56.
(9) Phan-Xuan, T.; Thuresson, A.; Skepö, M.; Labrador, A.; Bordes, R; Matic, A. Aggregation behavior of aqueous cellulose nanocrystals: the effect of inorganic salts. Cellulose 2016, 23 (6), 3653-3663.

(10) Xu, Y.; Atrens, A. D.; Stokes, J. R. Rheology and microstructure of aqueous suspensions of nanocrystalline cellulose rods. J. Colloid Interface Sci. 2017, 496, 130-140.

(11) Le Goff, K. J.; Gaillard, C.; Garnier, C.; Aubry, T. Electrostatically driven modulation of the reinforcement of agarose hydrogels by cellulose nanowhiskers. J. Appl. Polym. Sci. 2016, 133 (8), 43063.

(12) Martínez, D. G.; Stading, M.; Hermansson, A.-M. Viscoelasticity and microstructure of a hierarchical soft composite based on nanocellulose and $\kappa$-carrageenan. Rheol. Acta 2013, 52 (10), 823-831.

(13) McKee, J. R.; Hietala, S.; Seitsonen, J.; Laine, J.; Kontturi, E.; Ikkala, O. Thermoresponsive Nanocellulose Hydrogels with Tunable Mechanical Properties. ACS Macro Lett. 2014, 3 (3), 266-270.

(14) Dash, R.; Foston, M.; Ragauskas, A. J. Improving the mechanical and thermal properties of gelatin hydrogels cross-linked by cellulose nanowhiskers. Carbohydr. Polym. 2013, 91 (2), 638-645.

(15) De France, K. J.; Chan, K. J. W.; Cranston, E. D.; Hoare, T. Enhanced Mechanical Properties in Cellulose Nanocrystal-Poly(oligoethylene glycol methacrylate) Injectable Nanocomposite Hydrogels through Control of Physical and Chemical Cross-Linking. Biomacromolecules 2016, 17 (2), 649-660.

(16) Yang, D.; Peng, X.; Zhong, L.; Cao, X.; Chen, W.; Wang, S.; Liu, C.; Sun, R. Fabrication of a highly elastic nanocomposite hydrogel by surface modification of cellulose nanocrystals. RSC Adv. 2015, 5 (18), 13878-13885.

(17) Oguzlu, H.; Danumah, C.; Boluk, Y. The role of Dilute and Semi-dilute Cellulose Nanocrystal (CNC) Suspensions on the Rheology of Carboxymethyl Cellulose (CMC) solutions. Can. J. Chem. Eng. 2016, 94 (10), 1841-1847.

(18) Lee, Y. R.; Park, D.; Choi, S. K.; Kim, M.; Baek, H. S.; Nam, J.; Chung, C. B.; Osuji, C. O.; Kim, J. W. Smart Cellulose Nanofluids Produced by Tunable Hydrophobic Association of Polymer-Grafted Cellulose Nanocrystals. ACS Appl. Mater. Interfaces 2017, 9 (36), 31095-31101.

(19) Hasani, M.; Cranston, E. D.; Westman, G.; Gray, D. G. Cationic Surface Functionalization of Cellulose Nanocrystals. Soft Matter 2008, 4 (11), 2238-2244.

(20) Obiro, W. C.; Sinha Ray, S.; Emmambux, M. N. V-amylose Structural Characteristics, Methods of Preparation, Significance, and Potential Applications. Food Rev. Int. 2012, 28 (4), 412-438.

(21) Kaneko, Y.; Beppu, K.; Kadokawa, J.-i. Preparation of Amylose/ Polycarbonate Inclusion Complexes by Means of Vine-Twining Polymerization. Macromol. Chem. Phys. 2008, 209 (10), 1037-1042.

(22) Kaneko, Y.; Kadokawa, J.-i. Vine-twining polymerization: A new Preparation Method for Well-defined Supramolecules Composed of Amylose and Synthetic Polymers. Chem. Rec. 2005, 5 (1), 36-46.

(23) Fu, C.; Meng, L.; Lu, Q.; Zhang, X.; Gao, C. Large-Scale Production of Homogeneous Helical Amylose/SWNTs Complexes with Good Biocompatibility. Macromol. Rapid Commun. 2007, 28 (22), 2180-2184.

(24) Miles, M. J.; Morris, V. J.; Orford, P. D.; Ring, S. G. The Roles of Amylose and Amylopectin in the Gelation and Retrogradation of Starch. Carbohydr. Res. 1985, 135 (2), 271-281.

(25) Morris, V. J. Starch Gelation and Retrogradation. Trends Food Sci. Technol. 1990, 1, 2-6.

(26) Elazzouzi-Hafraoui, S.; Nishiyama, Y.; Putaux, J.-L.; Heux, L.; Dubreuil, F.; Rochas, C. The Shape and Size Distribution of Crystalline Nanoparticles Prepared by Acid Hydrolysis of Native Cellulose. Biomacromolecules 2008, 9 (1), 57-65.

(27) Salminen, R.; Baccile, N.; Reza, M.; Kontturi, E. Surface-Induced Frustration in Solid State Polymorphic Transition of Native Cellulose Nanocrystals. Biomacromolecules 2017, 18 (6), 1975-1982.

(28) Sirghi, L.; Kylián, O.; Gilliland, D.; Ceccone, G.; Rossi, F. Cleaning and Hydrophilization of Atomic Force Microscopy Silicon Probes. J. Phys. Chem. B 2006, 110 (51), 25975-25981. 